\title{
Direct temperature map estimation in optical long baseline interferometry
}

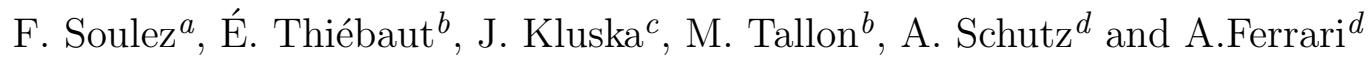 \\ ${ }^{a}$ Biomedical Imaging Group, École polytechnique fédérale de Lausanne, Lausanne CH-1015, \\ Switzerland. \\ ${ }^{b}$ Univ Lyon, Univ Lyon1, Ens de Lyon, CNRS, Centre de Recherche Astrophysique de Lyon \\ UMR5574, F-69230, Saint-Genis-Laval, France \\ ${ }^{c}$ University of Exeter Department of Physics and Astronomy Stocker Road, Exeter, Devon \\ EX4 4QL UK \\ ${ }^{d}$ Lab. J.-L. Lagrange, Université de Nice Sophia Antipolis, CNRS, Observatoire de la Côte \\ d'Azur, Parc Valrose, F-06108 Nice cedex 02, France
}

\begin{abstract}
We present a method to recontruct temperature map directly from spectro-interferometric data. It uses a sparse coding method to describe each pixel as a blackbody. Results are shown on the Herbig Be HD98922. Aside from recovering the mean environment temperature of $1654 \mathrm{~K}$ in agreement with the photometry, this technique allows us to study the temperature distribution in the first astronomical units around the star.
\end{abstract}

\section{INTRODUCTION}

The arrival on $2^{\text {nd }}$ generation beam combiners GRAVITY and MATISSE at the VLTI sheds light on the need of multispectral image reconstruction algorithm. Indeed, as they combine four telescopes with medium and high spectral resolution, it is now conceivable to use them as high spatial resolution integral field spectrograph by mean of image reconstruction. Image reconstruction is a very challenging problem. Recently, as part of the effort of the community to develop the research on multispectral image reconstruction the POLCA project gaves birth to three methods : MiRA3D,${ }^{1}$ PAINTER $^{2}$ and SPARCO. ${ }^{3}$

Spatio-spectral data cubes give very valuable astrophysical information such as velocity, abundance or temperature map. It is important to notice that this astrophysical information represents often few estimated parameters per pixel (e.g. dispersion and mean velocity, temperature, relative abundance of few chemical elements,...) and lies only on a complex but small subset of the spectrum. Using this fact as priors to better constraint the reconstruction, we propose a new algorithm that provides directly a 2D map of parameters of astrophysical interest. In interferometry, such an idea was already implemented using CLEAN in the OYSTER package. ${ }^{4}$ It is also behind the principle $\mathrm{SPARCO}^{3}$ method that only estimates from interferometric data a $2 \mathrm{D}$ image and a parametric model with different spectral indexes. In the presented work, we describe a method to reconstruct the temperature map from the polychromatic interferometric measurements.

\section{DICTIONARY BASED POLYCHROMATIC IMAGE RECONSTRUCTION}

The interferometric image reconstruction consists in recovering the polychromatic object $\boldsymbol{x}$ given the interferometric measurements (i.e. in most cases: squared visibilities and phase closures). This object is discretized as a vector of $K$ pixels and $L$ wavelengths: $\boldsymbol{x}=\left(w_{1,1}, \ldots, w_{K, L}\right)$. Most algorithm solves this problem as a constrained minimization:

$$
\boldsymbol{x}^{+}=\underset{\boldsymbol{x} \geq 0}{\arg \min }(\mathcal{L}(\boldsymbol{x})+\mu \mathcal{R}(\boldsymbol{x})) \text {, s.t. } \forall \ell, \sum_{k} x_{k, \ell}=1
$$

where $\mathcal{L}$ is a likelihood term ensuring that the model is in agreement with the data, $\mathcal{R}$ is a regularization function that enforces some priors and $\mu \geq 0$ an hyper-parameter. The constraints $\sum_{k} x_{k, \ell}=1$ ensure the spatial normalization of $\boldsymbol{x}$ as the visibilities are intrinsically normalized in each spectral channel $\ell$.

Optical and Infrared Interferometry and Imaging V, edited by Fabien Malbet,

(c) 2016 SPIE - CCC code: 0277-786X/16/\$18 - doi: 10.1117/12.2232577 


\begin{tabular}{ccc|l}
\hline Type & Distance & $T_{\text {eff }}$ & ref. \\
\hline B9Ve & $1150_{360}^{930}$ & $10500 \pm 500$ & Alecian et al. \\
A2III & 507 & 9000 & Hales et al. \\
\hline
\end{tabular}

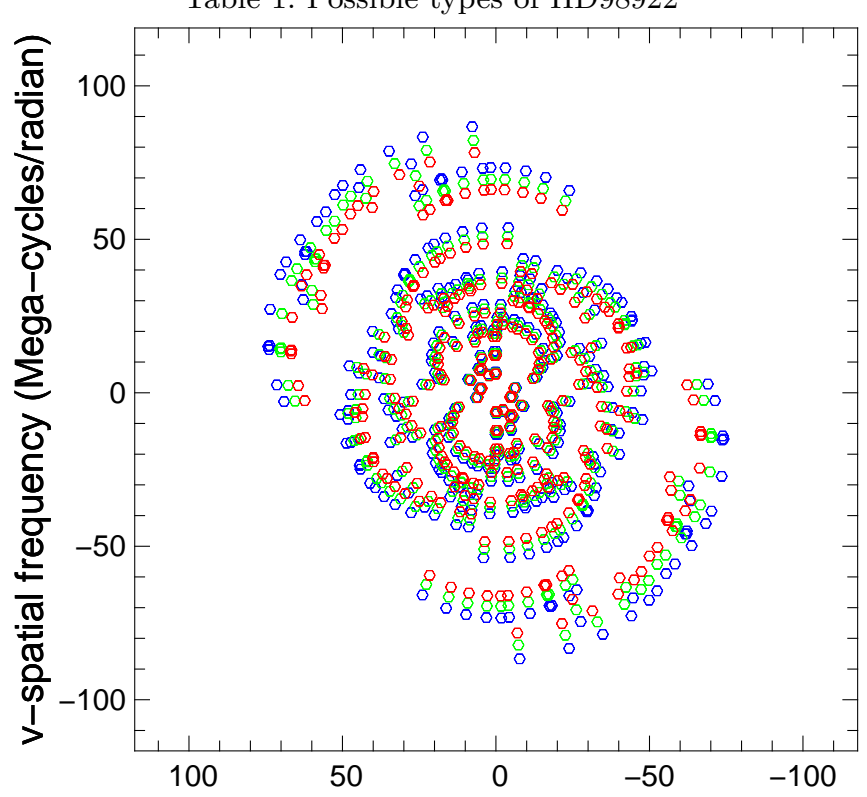

\section{u-spatial frequency (Mega-cycles/radian)}

Figure 1. $(u, v)$ coverage of the observations. The three colors correspond to the PIONIER spectral channels.

In our approach, we assume that the spectrum $\boldsymbol{x}_{k}$ of each pixel $k$ can be described by a linear combination of few spectra given by the dictionary $\mathbf{D}$ such that $\boldsymbol{x}_{k}=\mathbf{D} \boldsymbol{\alpha}$. In that case, the Equation 1 becomes a sparse coding problem: ${ }^{5}$

$$
\boldsymbol{x}^{+}=\underset{\boldsymbol{x}}{\arg \min }\left(\mathcal{L}(\boldsymbol{x})+\gamma \sum_{k}\left\|\boldsymbol{\alpha}_{k}\right\|_{1}\right) \text { s.t. }\left\{\begin{array}{l}
\forall \ell, \sum_{k} x_{k, \ell}=1 . \\
\boldsymbol{x} \geq 0 \\
\forall k, \boldsymbol{s} \times \boldsymbol{x}_{k}=\mathbf{D} \boldsymbol{\alpha}_{k},
\end{array}\right.
$$

The $\ell_{1}$ norm of $\boldsymbol{\alpha}_{k}$ acts as the regularization function $\mathcal{R}$ and is used to enforce its sparsity (i.e. most elements of $\boldsymbol{\alpha}_{k}$ are null). The level of sparsity is balanced by the hyper-parameter $\gamma \geq 0$. As all spectral channels are normalized, the information about the relative flux between each channel is lost. Hence, each spectrum has to be rescaled by the spectrum $s$ of the whole object before its decomposition on the dictionary. Following the framework developed for the MiRA3D ${ }^{1}$ and PAINTER ${ }^{2}$ algorithm, we solve Equation 2 using the Alternating Direction Method of Multipliers (ADMM).

\section{RESULTS ON HD98922}

Optical interferometry is the only mean to observe the central astronomical unit of the young objects. The study of the phenomena at this scale was the aim of a large observational program on 55 Herbig AeBe with VLTI/PIONIER conducted by J.-P. Berger in 2012/2013 (ID: 090.C-0963). ${ }^{8}$ Among these stars, the observation of HD98922 has one of the best $(u, v)$ coverage (see Figure 1). HD98922 is an interesting group II Herbig Be star having an SED suggesting a full disc without evidence for a gap. Its type is however not precisely known as indicated on Table 1. It was observed in the first two months of 2013 with the four $1.8 \mathrm{~m}$ Auxiliary Telescopes (ATs) of the Very Large Telescope using PIONIER ${ }^{9}$ (see table 3), with using the low spectral resolution in $\mathrm{H}$ band with 3 spectral channels ()$R \approx 15)$. 


\begin{tabular}{lllll}
\hline Obs. Date & Configuration & $\mathrm{MJD}$ & $\mathrm{nV}^{2}$ & $\mathrm{nCP}$ \\
\hline 2013-01-26 & A1-G1-J3-K0 & 56318.4 & 18 & 12 \\
2013-01-27 & A1-G1-J3-K0 & 56319.3 & 51 & 30 \\
2013-01-28 & A1-G1-J3-K0 & 56320.4 & 54 & 36 \\
2013-01-30 & A1-G1-J3-K0 & 56322.3 & 24 & 12 \\
$2013-01-31$ & A1-G1-J3-K0 & 56323.2 & 36 & 24 \\
$2013-02-01$ & A1-G1-J3-K0 & 56324.2 & 54 & 36 \\
$2013-02-17$ & D0-G1-H0-I1 & 56340.2 & 54 & 36 \\
$2013-02-18$ & D0-G1-H0-I1 & 56341.3 & 72 & 48 \\
$2013-02-19$ & D0-G1-H0-I1 & 56342.3 & 18 & 12 \\
$2013-02-20$ & D0-G1-H0-I1 & 56343.2 & 36 & 24 \\
\hline \multicolumn{5}{c}{ Table 2. HD98922 observation log. }
\end{tabular}

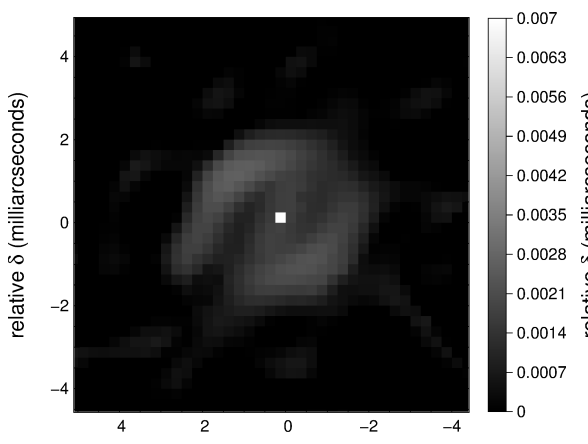

relative $\alpha$ (milliarcseconds)

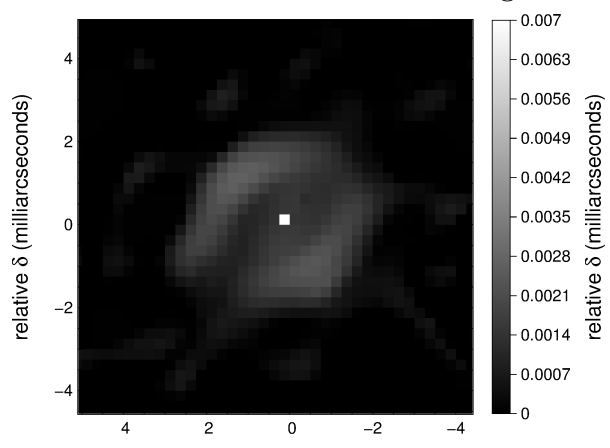

relative $\alpha$ (milliarcseconds)

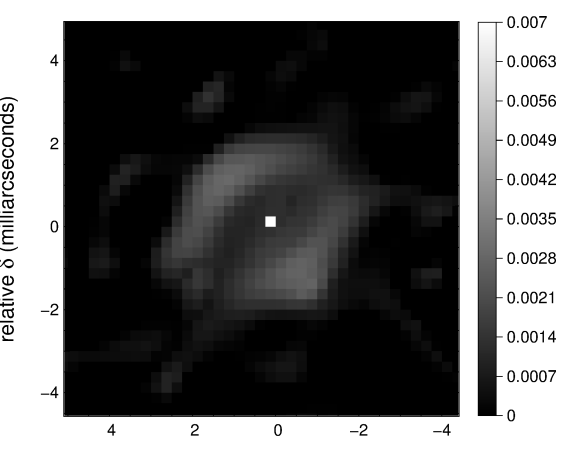

relative $\alpha$ (milliarcseconds)

Figure 2. $1.59 \mu \mathrm{m}$ channel of HD98922 Figure 3. $1.68 \mu \mathrm{m}$ channel of HD98922 Figure 4 . $1.77 \mu \mathrm{m}$ channel of HD98922 reconstruction

reconstruction

\subsection{Building up the dictionary}

We assume that the HD98922 SED is dominated by thermal emission and that its circumstellar disk can be modeled as a blackbody with spatially varying temperature. The dictionary $\mathbf{D}$ is thus built from a set of 500 spectra of black bodies ranging from $500 \mathrm{~K}$ to $20000 \mathrm{~K}$.

No simultaneous spectrum of HD98922 was available to set the scaling vector $\boldsymbol{s}$. To overcome that issue, we estimated the spectrum $s$ such as the star (i.e. central pixel) had the theoretical spectrum given by its spectral type. Although the type of HD98922 is not precisely known, both types indicated in Table 1 (B9V and A5III) have a quasi identical spectra in $\mathrm{H}$ band. Consequently, we have made a reconstruction using the Kurucz spectrum $^{10}$ for a B9V of $10500 \mathrm{~K}$ with $[\mathrm{Fe} / \mathrm{H}]=0$ and $\log g_{\star}=4$.

\subsection{Temperature map reconstruction}

A reconstruction (pixel size: 0.25 mas) was performed with $\gamma=10^{-6}$. After 200 iterations, the final reduced $\chi^{2}$ was $\approx 1.2$. The three reconstructed channels are shown Figure 2 to Figure 4 .

Due to the sparsity constraint, for each pixel $k$ the parameter $\boldsymbol{\alpha}_{\boldsymbol{k}}$ have only very few non-zero elements. In practice, we noticed that for each pixel $k$, only one element was significativelly non-zero. As each column of the dictionary $\mathbf{D}$ are spectra of black bodies, the index of this non-zero element of $\boldsymbol{\alpha}_{\boldsymbol{k}}$ gives directly a good estimate of the temperature of the object at pixel $k$. We are thus able to recover the temperature map in the first astronomical units around the star directly from the reconstruction without further processing. This temperature map is given on Figure 5. The disk parameters retrieved from this reconstruction are shown on Table 3.

\begin{tabular}{ccccc} 
diameter (major axis) & inclination & $\log \left(T_{\text {env }}\right)(\mathrm{K})$ & $T_{\text {env }}$ & disk relative flux \\
\hline 4.5 mas & $45^{\circ}$ & 3.218 & 1654 & 0.66 \\
& Table 3. Estimated parameters of the disk
\end{tabular}




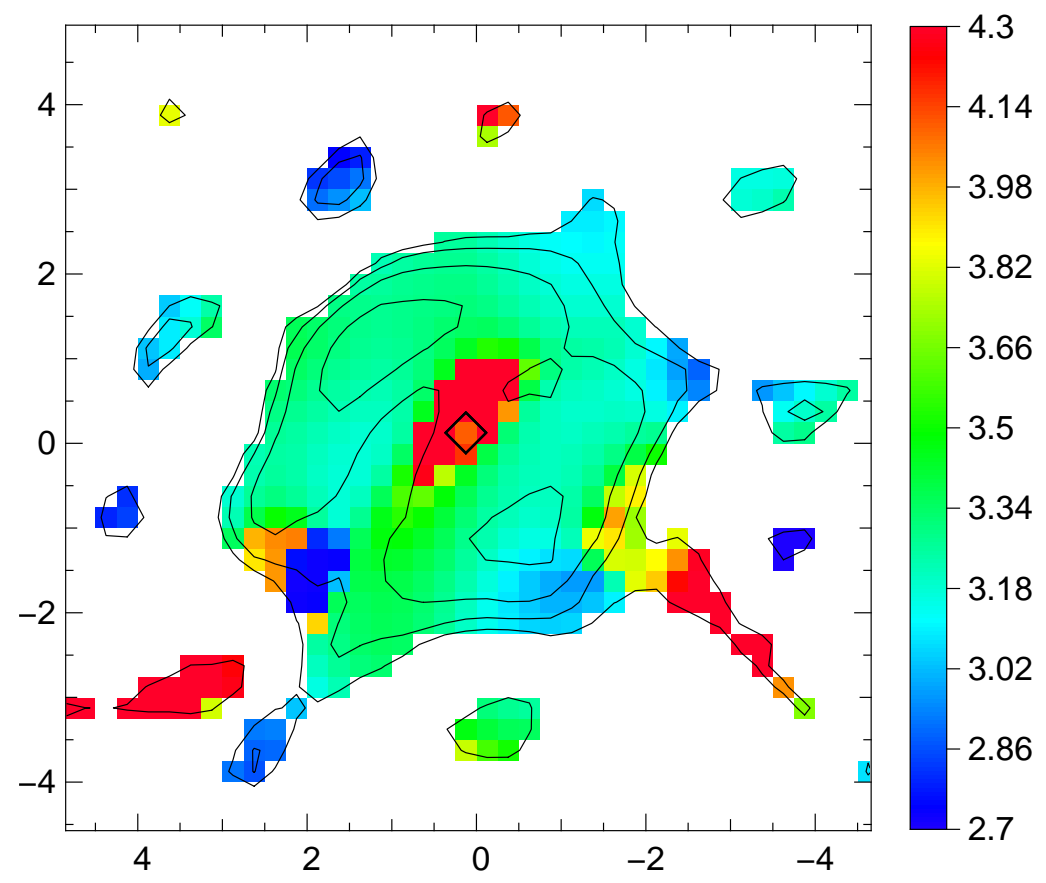

Figure 5. Recovered temperature map of HD98922

On the reconstructed image (Figure 2 to Figure 4) the disk is well resolved and its inner rim is clearly visible. From the temperature map on Figure 5 we estimated the relative flux of the disk of 0.66 and its mean temperature of $1654 \mathrm{~K}$. That is consistent with a relative flux of 0.66 and a temperature of $1493 \mathrm{~K}$ estimated from photometric measurements. ${ }^{8}$ In addition, some flux seems to be emitted from inside the cavity especially in the bluer part of the spectrum.

A reconstruction at such scale raises several new questions. Taking into account the geometry, one of the sides should be less bright. For the shortest wavelengths, it seems also that the flux emitted from inside the cavity may come from a gaseous internal disk.

\section{CONCLUSION}

This work illustrates perfectly the interest of the image reconstruction in interferometry for observing precisely the close environments of YSOs $(\approx 1 \mathrm{UA})$. It provides a new way to estimate quantities of astrophysical interest (here the temperature map) directly from the data. It can be viewed as a generalization of the SPARCO method ${ }^{3}$ with as much black-bodies as pixels. It is simpler and more robust than a classical image reconstruction followed by temperature estimation that can introduce additional errors and biases.

More generally it illustrates the potential of approaches based on dictionaries for all the hyperspectral instruments like integral-field spectrographs. Indeed, introducing into the dictionary spectra coming from different models will allow to rapidly discriminate the most probable model.

\section{Acknowledgements}

This work is supported by the Sinergia project "Euclid: precision cosmology in the dark sector" from the Swiss National Science Foundation and by the French ANR POLCA project (Processing of pOLychromatic interferometriC data for Astrophysics, ANR-10-BLAN-0511). 


\section{REFERENCES}

[1] Soulez, F. and Thiébaut, E., "An image reconstruction framework for polychromatic interferometry," in [Improving the performances of current optical interferometers, International Colloquium at haute provence observatory], . (2013).

[2] Schutz, A., Ferrari, A., Mary, D., Soulez, F., Thiébaut, É., and Vannier, M., "Painter: a spatiospectral image reconstruction algorithm for optical interferometry," J. Opt. Soc. Am. A 31(11), 2334-2345 (2014).

[3] Kluska, J., Malbet, F., Berger, J.-P., Baron, F., Lazareff, B., Le Bouquin, J.-B., Monnier, J., Soulez, F., and Thiébaut, E., "Sparco: a semi-parametric approach for image reconstruction of chromatic objectsapplication to young stellar objects," Astronomy \& Astrophysics 564, A80 (2014).

[4] Hummel, C. A., "A novel imaging algorithm for broadband aperture synthesis data," in [Optical and Infrared Interferometry II], 7734, 77342H (July 2010).

[5] Mairal, J., Bach, F., Ponce, J., and Sapiro, G., "Online dictionary learning for sparse coding," in [Proceedings of the 26th Annual International Conference on Machine Learning], 689-696, ACM (2009).

[6] Alecian, E., Wade, G., Catala, C., Grunhut, J., Landstreet, J., Bagnulo, S., Böhm, T., Folsom, C., Marsden, S., and Waite, I., "A high-resolution spectropolarimetric survey of herbig ae/be stars-i. observations and measurements," Monthly Notices of the Royal Astronomical Society 429(2), 1001-1026 (2013).

[7] Hales, A. S., De Gregorio-Monsalvo, I., Montesinos, B., Casassus, S., Dent, W. F. R., Dougados, C., Eiroa, C., Hughes, A. M., Garay, G., Mardones, D., Ménard, F., Palau, A., Pérez, S., Phillips, N., Torrelles, J. M., and Wilner, D., "A CO Survey in Planet-forming Disks: Characterizing the Gas Content in the Epoch of Planet Formation," Astron. J. 148, 47 (Sept. 2014).

[8] Lazareff, B., Berger, J.-P., Kluska, J., Bouquin, J.-B. L., Benisty, M., Malbet, F., Koen, C., Pinte, C., Thi, W.-F., Absil, O., Baron, F., Delboulbé, A., Duvert, G., Isella, A., Jocou, L., Juhasz, A., Kraus, S., Lachaume, R., Ménard, F., Millan-Gabet, R., Monnier, J., Perraut, K., Soulez, F., Tallon, M., Thiébaut, E., Traub, W., , and Zins, G., "Few puffed-up rims found in large interferometric survey of herbig aebe stars," Astron. ES Astrophys. (in prep).

[9] Le Bouquin, J.-B., Berger, J.-P., Lazareff, B., Zins, G., Haguenauer, P., Jocou, L., Kern, P., MillanGabet, R., Traub, W., Absil, O., et al., "Pionier: a 4-telescope visitor instrument at vlti," Astronomy \& Astrophysics 535, A67 (2011).

[10] Kurucz, R. L., "Model atmospheres for G, F, A, B, and O stars," Astrophys. J. Supp. 40, 1-340 (May 1979). 